\title{
Non-anomalous diffusion is not always Gaussian
}

\author{
Giuseppe Forte ${ }^{1}$, Fabio Cecconi ${ }^{2}$, Angelo Vulpiani ${ }^{1,3}$ \\ 1 Dipartimento di Fisica Università di Roma "Sapienza", Piazzale Aldo Moro 2, I-00185 Roma, Italy \\ ${ }^{2}$ CNR-Istituto dei Sistemi Complessi (ISC), Via dei Taurini 19, I-00185 Roma, Italy \\ 3 CNR-Istituto dei Sistemi Complessi (ISC), Piazzale Aldo Moro 2, I-00185 Roma, Italy.
}

\begin{abstract}
Through the analysis of unbiased random walks on fractal trees and continuous time random walks, we show that even if a process is characterized by a mean square displacement (MSD) growing linearly with time (standard behaviour) its diffusion properties can be not trivial. In particular, we show that the following scenarios are consistent with a linear increase of MSD with time: i) the highorder moments, $\left\langle[x(t)]^{q}\right\rangle$ for $q>2$ and the probability density of the process exhibit multiscaling; ii) the random walk on certain fractal graphs, with non integer spectral dimension, can display a fully standard diffusion; iii) positive order moments satisfying standard scaling do not imply an exact scaling property of the probability density.
\end{abstract}

\section{INTRODUCTION}

A colloidal particle in a fluid at thermal equilibrium undergoes a random displacement $x(t)$ due to the collisions with the surrounding molecules. Einstein [1] proved for the first time that the random variable $x(t)$ follows a Gaussian distribution at large times (see [2] and reference therein for a modern perspective). In particular, the mean square displacement (MSD) is proportional to the elapsed time, i.e.

$$
\left\langle x^{2}(t)\right\rangle \sim t
$$

$\langle\ldots\rangle$ being the ensemble average over a set of initial conditions $x_{i}(0)(i=1,2,3, \ldots)$. This scenario is referred to as normal diffusion and it is a directed consequence of the Central Limit Theorem (CLT), as the total displacement of a given particle at time $t$

$$
x_{i}(t)=\sum_{s=0}^{t} \Delta x_{i}(s)
$$

is the sum over the elementary displacements, $\Delta x_{i}(s)=$ $x_{i}(s)-x_{i}(s-1)$. A classical result of the probability theory states that if $\Delta x_{i}(s)$ are independent (or weakly dependent) variables then $x_{i}(t)$ is a Gaussian-distributed variable at large $t$.

It is easy to show that a linear behaviour of the MSD is not a prerogative of Gaussian distribution only, in fact every distribution with a self similar asymptotic property

$$
P(x, t) \sim \frac{1}{\lambda(t)} f\left(\frac{x}{\lambda(t)}\right)
$$

satisfies the condition $\left\langle x^{2}(t)\right\rangle \sim t$, upon choosing the lengthscale such that $\lambda(t) \sim t^{1 / 2}$. Therefore, the knowledge of $\left\langle x^{2}(t)\right\rangle$ alone is poorly informative and its linear behaviour is not sufficient to assess neither that the diffusion is standard nor that the corresponding distribution $P(x, t)$ is Gaussian, see Ref. [3] for a nice and interesting analysis.

Moreover, deviations from the standard behavior are now well known and frequently observed in experiments, computer simulations, natural, economic and social processes [4]. Such deviations are classified as anomalous diffusion

$$
\left\langle x^{2}(t)\right\rangle \sim t^{2 \nu}
$$

with $\nu \neq 1 / 2[5]$ ] $]$. The case $\nu<1 / 2$ is called subdiffusion whereas $\nu>1 / 2$ is known as enhanced diffusion or superdiffusion. For a process characterized by anomalous diffusion, the simplest scenario that can occur is a corresponding distribution which, for enough large $t$, still satisfies Eq. (11) with $\lambda(t) \sim t^{\nu / 2}$.

The self similar scaling (11) of the PdF automatically establishes the specific relationship

$$
\left\langle|x(t)|^{q}\right\rangle=\left\langle x^{2}(t)\right\rangle^{q / 2} \sim \lambda(t)^{q / 2} .
$$

among the moments in both anomalous and standard regimes.

More interesting situations occur when the property (3) is violated and it is replaced by the more general behaviour

$$
\left\langle|x(t)|^{q}\right\rangle \sim t^{q \nu(q)}
$$

where $q \nu(q)$ is a nonlinear function of $q$. This is the case of a superdiffusive process that could be affected by the so-called strong anomalous diffusion [8, 9]. The property (44) is generally referred in the literature to as multiscaling [10 12] in order to distinguish it from the ordinary or simple scaling characterized by self similarity, Eq. (11).

Indeed, the presence of a non-constant spectrum of exponents $\nu(q)$ for the time behaviour of the moments implies that the self similarity (1) fails, so there is no chance to have a unique collapse of the PdFs at different times onto a single curve. The simplest, yet not unique possibility, occurs when the bulk and the tails of a PdF satisfy a different scaling law thus undergoing two separate collapses.

In this paper, we are interested in discussing through examples how the scaling behaviour (11) can be satisfied or violated. This issue becomes rather crucial in singleparticle tracking experiments [7, 13], when from a long but finite dataset of position measurements one would 
infer the statistical properties of the underlying particle dynamics.

Specifically, we will show that even in the apparently safe cases where $\nu(2)=1 / 2$, a non Gaussian PdF is possible with anomalous scaling of moments of order $q>2$, in particular $\left\langle x^{4}(t)\right\rangle \sim t^{4 \nu(4)}$ with $4 \nu(4)>2$. Conversely, there are cases with a standard scaling of moments without a collapse of the PdF. Actually this is not surprising, as the knowledge of all the positive integer moments is not fully equivalent to the knowledge of the PdF, indeed examples can be constructed where two different distributions share the same moments. Hence the reconstruction of the probability distribution from the sequence, $\mu_{k}=\left\langle x^{k}\right\rangle(k=0,1,2, \ldots, \infty)$, of positive integer moments is a classical and delicate issue of statistical mathematics, known as the problem of moments, which was formulated by T. Stieltjes in 1894 [14]. A general solution was given by Carleman [15] who identified a sufficient condition for which a probability distribution is uniquely determined by its infinite sequence of positive integer moments.

The paper is organized as follows: in Sec. 2, we revisit the continuous time random walk as an example of strong anomalous behaviour showing that, despite the condition $\nu(2)=1 / 2$, its $\mathrm{PdF}$ has a multiscaling structure and $\nu(4)>1 / 2$. In Sec. 3 and Sec. 4 , we study the properties of the random walk on two fractal-like tree structures called in the following Nice Tree of dimension $k\left(N T_{k}\right)$ and Super Nice Tree $(S N T)$ respectively.

The random walk on both graphs exhibits an ordinary scaling of moments, however, while in the $N T_{k}$ we find also a self similar scaling of the PdF, the scaling of the $\mathrm{PdF}$ in the $S N T$ structure is violated at small arguments. Conclusions are found in Sec.5.

\section{THE DIFFUSIVE PROPERTIES OF THE CONTINUOUS TIME RANDOM WALK}

Continuous time random walk (CTRW) was introduced by Montroll and Weiss, in a series of pioneering papers on the diffusion processes on lattices, see e.g. [16, 17]. We consider here a CTRW variant known as velocity model [18], where a particle undergoes a series of kicks (collisions) at random times $t_{1}, t_{1}, \ldots, t_{n}, \ldots$ and between two consecutive collisions its velocity remains constant. The position of the particle at time $t$, such that $t_{n}<t \leq t_{n+1}$, will be

$$
x(t)=x\left(t_{n}\right)+v\left(t_{n}\right)\left(t-t_{n}\right)
$$

the time intervals $\tau_{n}=t_{n+1}-t_{n}$ are independent random variables with a truncated power law distribution

$$
P(\tau) \propto \begin{cases}\tau^{-g} & 1 \leq \tau \leq T \\ 0 & \text { elsewhere }\end{cases}
$$

with $g>1$ and $v_{n}= \pm 1$ with equal probability. The lower cutoff $t_{c}=1$ is a regolarization to avoid the singularity from infinitesimally short steps, moreover we also introduce an upper cutoff $T$ whose technical utility will be clear below. The presence of cutoff $T$ implies that the hypothesis of the CLT for the process (5) are fulfilled, thus as $t \gg T$, it converges to a Gaussian process. However if $T$ is chosen sufficiently large [19], this convergence can be made slow enough that a long and robust preasymptotic regime of strong anomalous diffusion can be clearly observed. A quantity that will be important in the following is the $q$-order moment of the waiting time $\tau$ whose asymptotic scaling for large $T$ is the following

$$
\left\langle\tau^{q}\right\rangle_{c} \sim \begin{cases}T^{1-g+q} & \text { if } q>g-1 \\ a(q, g) & \text { if } q<g-1\end{cases}
$$

where $a(q, g)$ is a constant independent of $T$ and the index $c$ indicates the average over the "truncated" distribution.

At $T \rightarrow \infty$, various diffusive regimes occur depending on the value of the exponent $g$, see Andersen et al. [9]; in particular the case $g \in(3,4]$ corresponds to the anomalous diffusion, $\left\langle|x(t)|^{q}\right\rangle \sim t^{q \nu(q)}$, with

$$
q \nu(q)= \begin{cases}q / 2, & q=1,2 \\ q+2-g, & q=3,4,5, \cdots\end{cases}
$$

The above behaviour of $q \nu(q)$ is quite peculiar as it coincides with that one of standard diffusion for $q<2$, while for larger $q$, we have $q \nu(q) \neq q / 2$, and this represents an example of strong anomalous regime.

Following the reasoning of Andersen and coworkers [9], we compute the $q$-order moments of the variable

$$
x(t)=\sum_{i=1}^{n_{t}} v_{i} \tau_{i}
$$

at different $q$, where $n_{t}$ is the stochastic process counting the number of "collisions" (flights) the particle underwent within the time $t$. Odd-order moments $\left\langle[x(t)]^{q}\right\rangle$ vanishes for the symmetry $v \rightarrow-v$ of the velocity distribution. Even-order moments are nonzero and can be evaluated exploiting the following properties:

$\left\langle v_{i} \tau_{j}\right\rangle=0,\left\langle v_{i} v_{j}\right\rangle=\delta_{i j},\left\langle\tau_{i} \tau_{j}\right\rangle=\left\langle\tau^{2}\right\rangle \delta_{i j}$. The lowest non-zero moments of $x(t)$

$$
\left\langle x^{q}(t)\right\rangle=\left\langle\left(\sum_{i=1}^{N} v_{i} \tau_{i}\right)^{q}\right\rangle
$$

can be explicitly derived from the general multinomial formula

$$
\left\langle x^{q}(t)\right\rangle=\sum_{\{\mathbf{k}\}} \frac{q !}{k_{1} ! k_{2} ! \ldots k_{N} !} \prod_{j=1}^{N}\left\langle\left(v_{j} \tau_{j}\right)^{k_{j}}\right\rangle
$$

with $\{\mathbf{k}\}=\left\{k_{1} \ldots k_{N}\right\}$ indicating the sets of nonnegative even integer arrays such that $k_{1}+k_{2}+\ldots+k_{N}=$ $q$. 


\begin{tabular}{lc}
$\mathrm{q}$ & Moment \\
\hline \hline 2 & $N\left\langle\tau^{2}\right\rangle$ \\
4 & $N\left\langle\tau^{4}\right\rangle+3 N(N-1)\left\langle\tau^{2}\right\rangle^{2}$ \\
6 & $N\left\langle\tau^{6}\right\rangle+15 N(N-1)\left\langle\tau^{2}\right\rangle\left\langle\tau^{4}\right\rangle+15 N(N-1)(N-2)\left\langle\tau^{2}\right\rangle^{3}$ \\
8 & $N\left\langle\tau^{8}\right\rangle+28 N(N-1)\left\langle\tau^{6}\right\rangle\left\langle\tau^{2}\right\rangle+35 N(N-1)\left\langle\tau^{4}\right\rangle^{2}+210 N(N-1)(N-2)\left\langle\tau^{2}\right\rangle^{2}\left\langle\tau^{4}\right\rangle+105 N(N-1)(N-2)(N-3)\left\langle\tau^{2}\right\rangle^{4}$
\end{tabular}

TABLE I. Lowest four non-vanishing moments of the displacement Eq. (9)

In the above expression, we have implicitly taken the average $\left\langle n_{t}\right\rangle=N$ and exploited independence of the variables. In Table I, the explicit expressions of the lowest four non-vanishing moments are reported. With simple considerations it is clear what are the terms of the expansion which maximally contribute to the moments. First we have to observe that, when the time $t$ is so large that enough collisions (steps) occurred, $n_{t} \gg 1$, we have in a good approximation $t \simeq \sum_{i=1}^{n_{t}} \tau_{i}$, therefore as a consequence of the Law of Large Numbers, $t \simeq N\langle\tau\rangle_{c}$, where $N=\left\langle n_{t}\right\rangle$ is mean value of the number of time steps necessary to reach the time $t$. Accordingly for large times we can replace in all the formulas $N \sim t /\langle\tau\rangle_{c}$, with $g$ such that $\langle\tau\rangle_{c}$ is finite as $T \rightarrow \infty$. Now two regimes have to be considered depending on the ratio $t / T$. For $t / T \ll 1$ (i.e. $t$ in the anomalous regime), the $q$-order moment is dominated by $N\left\langle\tau^{q}\right\rangle_{c}$, the largest term in $T$, then

$$
\left\langle x^{q}(t)\right\rangle \sim t \frac{\left\langle\tau^{q}\right\rangle_{c}}{\langle\tau\rangle_{c}} .
$$

Whereas, when $t / T \gg 1$ ( $t$ in the Gaussian regime)

$$
\left\langle x^{q}(t)\right\rangle \sim t^{q}\left(\frac{\left\langle\tau^{2}\right\rangle_{c}}{\langle\tau\rangle_{c}}\right)^{q} .
$$

finally, at $t \sim T$ there is the crossover from the anomalous to the standard behaviour. The exponent $q \nu(q)$ is determined by matching, at the cutoff $t \sim T$, the anomalous scaling of the q-moments $T^{q \nu(q)}$ with the scaling of the most divergent term in expansion (10), $T\left\langle\tau^{q}\right\rangle_{c} /\langle\tau\rangle_{c}$

$$
T^{q \nu(q)} \sim T \times T^{1-g+q}
$$

from which $q \nu(q)=2-g+q$ [see Eq. (8)] ].

The behaviour $\left\langle|x(t)|^{q}\right\rangle \sim t^{q \nu(q)}$ of the CTRW moments is verified by simulating $6.4 \times 10^{7}$ independent CTRW trajectories with $g=3.2$ and computing the ensemble average $\langle\cdots\rangle$ at different times. The results are reported in Fig. 1]. The inset shows the nonlinearity of the exponent $q \nu(q)$ as a function of $q$ proving the multiscaling character of the diffusion.

The relation (8) provides strong indications on the possible form of the PdF; the lowest order moments behave in time as in the case of normal diffusion, thus we expect that the $P(x, t)$ has a Gaussian bulk which scales as $\left.P(x, t)=t^{-1 / 2} f\left(x / t^{1 / 2}\right)\right)$, with $f(u)=$ $\left(2 \pi \sigma^{2}\right)^{-1 / 2} \exp \left[-u^{2} /\left(2 \sigma^{2}\right)\right]$ for moderate value of the argument $|u / \sigma|$. In the range $\sigma \sqrt{t} \ll x \lesssim c \sim t$ the behaviour of the high order moments suggests the following

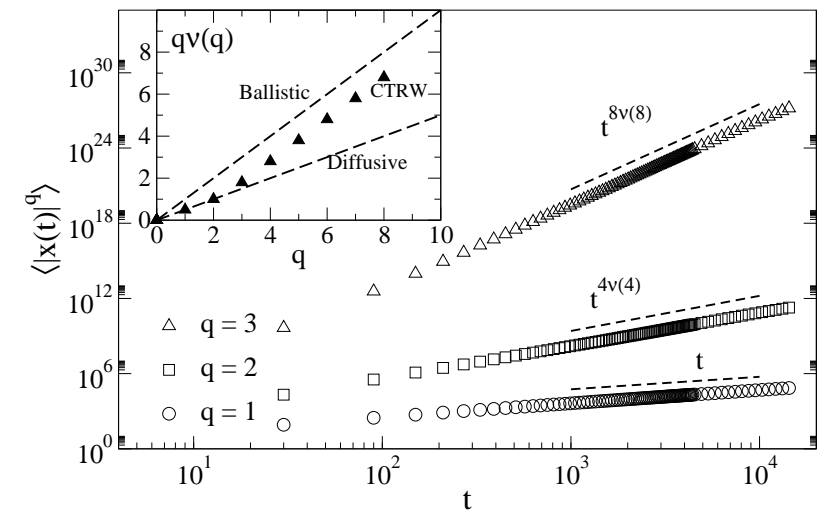

FIG. $1 .\left\langle x^{q}(t)\right\rangle$ vs $t(q=2,4,8)$ resulting by a simulation of $6.4 \times 10^{7}$ CTRW realizations (5) with $g=3.2$; the inset shows the relation $q \nu(q)$ vs $q$ which underlines the strong anomalous diffusive character of this particular system.

form of the PdF

$$
P(x, t)= \begin{cases}\frac{1}{t^{1 / 2}} f\left(\frac{x}{t^{1 / 2}}\right), & x \leq c \sim t \\ 0, & x>c .\end{cases}
$$

The assumption (11) is consistent with Eq. (8) only if $x$ around $c \sim t$, the function $f$ is such that

$$
f\left(\frac{x}{t^{1 / 2}}\right) \sim\left(\frac{x}{t^{1 / 2}}\right)^{-\alpha}
$$

namely, the tails decay as power-law behavior with an exponent $\alpha$ related to $g$. Let $x^{*} \sim \sigma \sqrt{t}$ denote the value at which the crossover between

$$
\begin{array}{r}
\left\langle x^{q}(t)\right\rangle=\int_{0}^{\tilde{x}} d x x^{q} P_{t}(x) \sim \\
\int_{0}^{x^{*}} d x \frac{x^{q}}{t^{1 / 2}} f\left(\frac{x}{t^{1 / 2}}\right)+\operatorname{cost} \int_{x^{*}}^{c} d x \frac{x^{q}}{t^{1 / 2}}\left(\frac{x}{t^{1 / 2}}\right)^{-\alpha}
\end{array}
$$

the first term behaves as $t^{q / 2}$, whereas the second one behaves as $t^{q+\frac{1}{2}-\frac{\alpha}{2}}$. Therefore for small $q$ the dominant contribution comes from the first term, $\left\langle x^{q}(t)\right\rangle \sim t^{q / 2}$, while for large $q$ the leading contribution is given by the second term. The scaling exponent $q+(1-\alpha) / 2$ is in agreement with $q \nu(q)=q+2-g$, second line of Eq. (8), only if $\alpha=2 g-3$ which is the exponent of the expected power-law behavior of tails outside the Gaussian bulk.

The collapse of the rescaled PdF of the CTRW (5D) at different times is shown in Fig. 2. As it discussed in the 


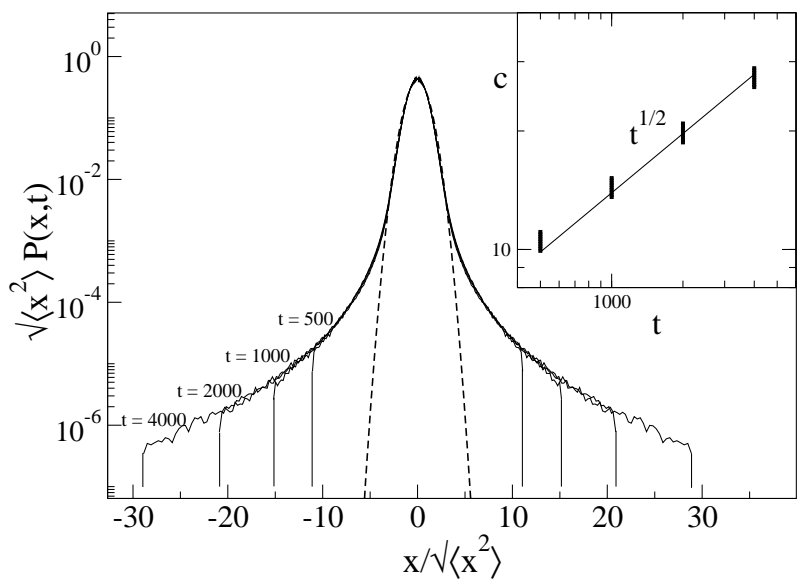

FIG. 2. Rescaled probability densities of the CTRW at different times for $g=3.2$. The PdF is obtained from the histogram over $6.4 \times 10^{7} \mathrm{CTRW}$ positions at times $t=$ $500,1000,2000,4000$. The vertical lines are guide for the eyes to mark the bounded support, $|x| \leq c(t)$, of the distributions according to Eq. (11), the dashed line represents the Gaussian PdF. The inset shows the scaling $c(t) / \sqrt{t} \sim t^{1 / 2}$ in Eq. (11).

above argument, the PdF must have a bounded support, $|x| \lesssim c(t)$ see Eq. (11).

It is worth remarking that the above result is not actually a violation of the Central Limit Theorem (CLT), as in the bulk all the PdFs collapse onto a Gaussian and only the far tails deviate from this behavior. The CLT, indeed, does not grant anything on the nature of the tails, it only specifies the shape of the limit distribution within the scaling region, $|x(t) / \sqrt{t}| \sim O(1)$. The tails outside such a bound are not universal and generally not Gaussian. Analogously, there is no reason for the high order moments, which receive the main contribution from tails, to converge to the Gaussian moments.

We conclude this section with a methodological comment. The statistical properties of the CTRW like moments or PdF at a certain time $t$ have been computed by following an ensemble of walkers up to $t$. However we checked that results obtained from the ensemble of trajectories are perfectly consistent with those obtained using a long single-realization [20]. The discrepancy between ensemble and single-trajectory analysis has important physical implications as it may indicate deviations from standard Brownian diffusion because of ergodicity breaking and aging in the process dynamics 21]. Here, we only mention this crucial issue while referring the reader to the works by Sokolov [20] and Barkai et al. 22] for a plain and nice discussion. However we stress that the considered velocity CTRW, for $g>3$ has a finite average time $\langle t\rangle$ and the presence of a characteristic time scale in the dynamics excludes ambiguous results from the single trajectory analysis and grants the equivalence between ensemble and running averages.

\section{DIFFUSION ON BRANCHED GRAPHS}

A undirected graph is a collection of vertices pairwise connected, or not, by links. To each graphs of $N$-vertices, we can associate a $N \times N$ matrix A (adjacency matrix), such that, $\mathrm{A}_{i j}=1$ if there is an link between vertices $i$ and $j, \mathrm{~A}_{i j}=0$ otherwise.

An unbiased random walk on a graph can be defined in a natural way: a walker at time $t$ on the node $i$ can jump at time $t+1$ on the node $j$ only if $\mathrm{A}_{i j}=1$, with a transition probability $P\{i \rightarrow j\}=1 / n_{i}$, where $n_{i}=$ $\sum_{j} \mathrm{~A}_{i j}$ is the number of links established by the node $i$. Then the diffusing variable is the position of the walker which takes on discrete values defined by the vertices of the graph. The latter assumption of equal-probability of the transition to nearest neighbors of $i$ can be relaxed.

The diffusion properties of a random walk on a graphs depend on both the fractal dimension 23] $d_{f}$ and the spectral dimension $d_{s}[24]$. The fractal dimension is related to the scaling of the number of points in a sphere of radius $\ell: N(\ell) \sim \ell^{d_{f}}$; the spectral dimension is defined by the return probability $P_{t}(x)$ to a generic site $x$ in $t$ steps $P_{t}(x) \sim t^{-d_{s} / 2}$. The ratio between $d_{s}$ and $d_{f}$ determines the mean square displacement through the relation [25]

$$
\left\langle x^{2}(t)\right\rangle \sim t^{d_{s} / d_{f}} .
$$

Analogously to the CTRW, we can investigate the behaviour of high-order moments and the possible collapse of the PdF. In particular, we focus on the random walks on a class of graphs the Nice Trees of dimension $k\left(N T_{k}\right)$, that are recursive fractal trees with the remarkable property that

$$
d_{f}=d_{s}(k)=1+\frac{\ln k}{\ln 2},
$$

i.e. the fractal and the spectral dimension coincide [26, 27] for any value of $k$. Hence, despite their fractallike structure, Eq. (12) prescribes a standard diffusion of the random walks on any $N T_{k}$. A $N T_{k}$ graph is defined recursively as follows. An origin $\mathcal{O}$ is connected with a point $A$ by a link of length 1 ; from $A$ the tree splits in $k$ branches of length $2^{1}$ each. The end point of such branches, in turn, splits again into $k$ branches of length $2^{2}$ and so on (see Fig. 3).

From the previous section, we learnt that a linear growth of the MSD in time (i.e. $2 \nu(2)=1$ ) does not grant a Gaussian diffusive process thus, we can wonder about the consequences of the property (13) on the behaviour of the full spectrum of moments and the PdF. In order to characterize the diffusion properties of the unbiased random walk on the $N T_{k}$, we need to numerically study the corresponding master equation of the process.

We can assign to each site the integer distance $x$, if it is connected to the origin $\mathcal{O}$ by the minimal path with $x$-links. Each sites of the $N T_{k}$ graph can be identified by a couple of indices $(x, \alpha)$ indicating the distance from $\mathcal{O}$ (depth of the tree) and the corresponding branch (Fig. 31). 


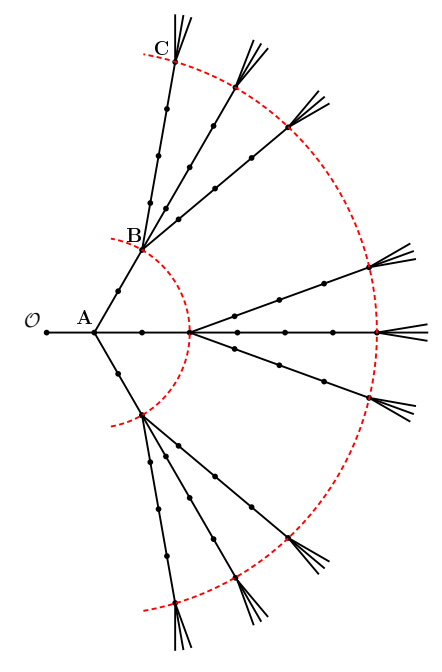

FIG. 3. Geometrical construction of a Nice Tree of dimension $k=3$. An origin $\mathcal{O}$ is connected with a point $A$ by a link of length 1 ; from $A$ the tree splits in $k$ branches of length $2^{1}$ each. The end point of each branch, in turn, splits again into $k$ branches of length $2^{2}$. The procedure is recursively iterated.

The structure of the master equation governing the evolution of the probability $Q_{t}(x, \alpha)$ that a walker occupies at time t the state $(x, \alpha)$ is understood by considering the possible transitions around a generic branching point (Fig. (4). The equation involves nearest neighbour sites $\{x-1, x, x+1\}$ which can belong to either the same branch or different but consecutive branches. Formally, it can be written as

$$
\begin{aligned}
Q_{t+1}(x, \alpha) & =W(x, \alpha \mid x-1, \alpha) Q_{t}(x-1, \alpha) \\
& +\sum_{\beta} W(x, \alpha \mid x+1, \beta) Q_{t}(x+1, \beta)
\end{aligned}
$$

with $\alpha, \beta$ identifying two consecutive branches and $W(x, \alpha \mid x-1, \alpha), W(x, \alpha \mid x+1, \beta)$ are the corresponding transition probabilities. As far as we are interested in the evolution of $P_{t}(x)$, the probability for the walker to be at distance $x$ at time $t$, we need to sum over the index $\alpha$, namely over all those branches containing a site at distance $x$ from the origin, then $P_{t}(x)=\sum_{\alpha} Q_{t}(x, \alpha)$. We can now consider the one-dimensional master equation obtained by "projecting" the complete master equation of the $N T_{k}$ onto the one-dimensional lattice $x \in\{n\}_{n=0}^{\infty}$. The procedure is a generalization of that used in Ref. [28] and with reference to Fig. [4 it leads to the following three cases

$$
\begin{aligned}
P_{t+1}(x-1) & =\frac{1}{2} P_{t}(x-2)+\frac{1}{k+1} P_{t}(x) \\
P_{t+1}(x) & =\frac{1}{2} P_{t}(x-1)+\frac{1}{2} P_{t}(x+1) \\
P_{t+1}(x+1) & =\frac{k}{k+1} P_{t}(x)+\frac{1}{2} P_{t}(x+2)
\end{aligned}
$$

where the first and third equations holds only for branching points $\left(x=2^{n}-1\right)$, the second one for all the other

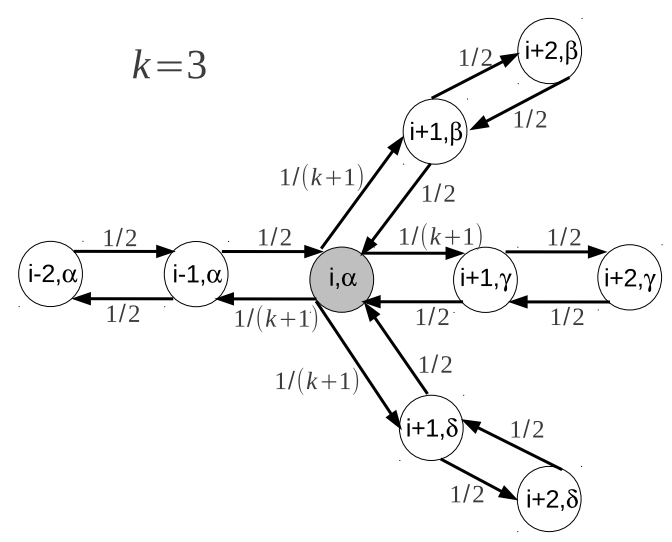

FIG. 4. Transitions around a branching point of a $N T_{k}$ with $k=3$ that have to be considered for the construction of the one-dimensional diffusion model. A walker on a branching point (shaded circle) has $k$ possibilities to make one step away from the origin and 1 possibility to get one step closer.

sites. Accordingly, the transition matrix $w(x \pm 1 \mid x)$ of the one-dimensional RW from $x \rightarrow x \pm 1$, in a time step reads

$$
\begin{aligned}
& w(x+1 \mid x)= \begin{cases}\frac{k}{k+1}, & \text { if } x=2^{n}-1 \\
1 / 2, & \text { elsewhere }\end{cases} \\
& w(x-1 \mid x)= \begin{cases}\frac{1}{k+1}, & \text { if } x=2^{n}-1 \\
1 / 2, & \text { elsewhere }\end{cases}
\end{aligned}
$$

where $2^{n}-1$ is the formula identifying the distance of the branching points from the origin $\mathcal{O}, W(1 \mid 0)=1$ is the condition for reflecting boundary in $\mathcal{O}$. The RW on $\mathrm{NT}_{k}$ is thus mapped onto a RW on a one-dimensional lattice in a deterministic heterogeneous environment. The physical interpretation of the transition matrix is simple, if a walker sits on a branching point, there are $k$ possibilities to go one step away from origin and 1 possibility to make one step closer. Then the next step will take it either farer from the origin with probability $p_{+}=k /(k+1)$ or closer the origin with probability $p_{-}=1 /(k+1)$. Whereas if the a walker is away from the branching point, both steps are unbiased, $p_{-}=p_{-}=1 / 2$. The inhomogeneity stems from the branching points $x=2^{n}-1, n=1,2,3 \cdots$ which represent special points ("defects") but become exponentially rare as long as the walker lies far away from the origin. Thus, far away from the origin, the process remains an unbiased RW for so long time that "Gaussian character" of the distribution is not altered by the presence defects.

It is well known that a coarse-graining procedure over a Markov process generally spoils the Markov property. However as a consequence of the peculiar structure of the transition probabilities the reduction to the single "radial 
coordinate" of Eq. (14) produces again a Markovian master equation.

The analytical solution $P_{t}(x)$ to master equation (15) cannot be derived in a simple explicit form, however it can be easily obtained by iterating numerically Eq. (15) from an initial condition.

As a first check of convergence of the numerical implementation of the 1D approach, we compute the return probability $P_{t}(\mathcal{O})$ to the origin in $t$ steps. Graph theory [25] predicts the power-law decay

$$
P_{t}(\mathcal{O}) \sim t^{-d_{s}(k) / 2}
$$

with $d_{s}(k)$ from Eq. (13), data of Fig. 5 perfectly verified the prediction. Figure 6 shows the simulation results for

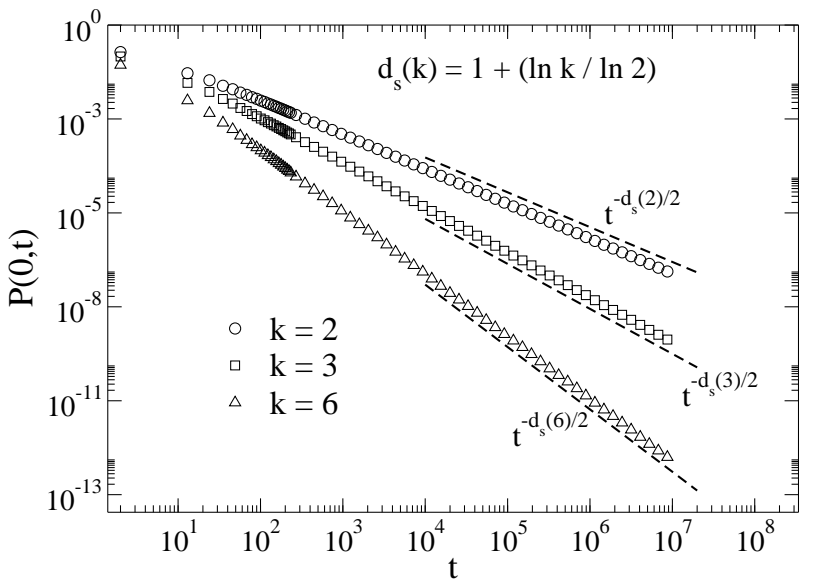

FIG. 5. Log-Log plot of the return probability to the origin in $t$ steps, $P_{t}(\mathcal{O})$ as a function of $t$, for $N T_{k}$ of dimension $k=2$ (circles), $k=3$ (squares) and $k=6$ (triangles). Data are in agreement with the Graph-Theory prediction $P_{t}(\mathcal{O}) \sim t^{-d_{s} / 2}$ with $d_{s}=1+\ln (k) / \ln (2)$, Eq. (13), dashed lines.

the probability density $P_{t}(x)$ rescaled to $x \rightarrow x / \sqrt{\left\langle x^{2}\right\rangle}$ and $P_{t}(x) \rightarrow \sqrt{\left\langle x^{2}\right\rangle} P_{t}(x)$ obtained by iterating the master Eq. (15) for a $\mathrm{NT}_{k}$ with $k=2$. The discontinuities of $P_{t}(x)$ are a clear signature of the "defects" (branching points on the tree) which interrupt the standard random walk in the passage from a branch to the successive one. The dash-dotted curve is the approximated solution

$$
F_{t}(x)=\frac{2 x^{d_{s}-1}}{\Gamma\left(d_{s} / 2\right)(2 t)^{d_{s} / 2}} \exp \left(-x^{2} / 2 t\right)
$$

which well interpolates the exact numerical result. Expression (18) is the radial Gaussian distribution in dimension $d_{s}(k)$ and it can be explained by the following heuristic argument. Let $\tilde{P}_{t} \sim \exp \left(-x^{2} / 2 t\right)$ be the probability density at time $t$ of an unbiased one-dimensional RW from zero to infinity. In a first approximation, $P_{t}(x)$ can be assumed as the product $N_{x} \tilde{P}_{t}(x)$, where $N_{x}$ is the number of sites at the same distance $x$ from the origin; we can write $N_{x}$ as $k^{n(x)}$, with $n(x)$ the number of branching points along a minimal-length path connecting $\mathcal{O}$ and $x$. The number $n(x)$ can be obtained

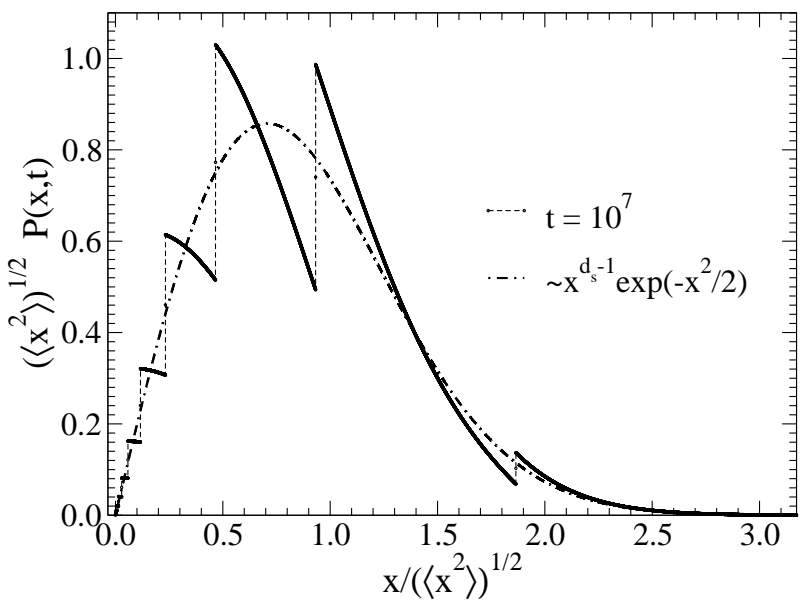

FIG. 6. Probability density obtained by simulating the master equation (15) referring to a $\mathrm{NT}_{k}$ with $k=2$. Dashed line indicates the analytical interpolation (18).

by observing that the branching points are those located at $x_{b r}=2^{n}-1(n=1,2,3, \cdots)$, from which $n(x)=\lfloor\ln (x+1) / \ln 2\rfloor$, so a given walker at distance $x$ has crossed $n(x) \approx \ln (x+1) / \ln 2$ possible ramification points. Now with the aid of Eq. (13), we can rewrite $N_{x} \sim x^{d_{s}(k)-1}$, which after normalization yields expression (18).

Using the approximation $F_{t}(x)$, we can estimate all the moments $\left\langle x^{q}(t)\right\rangle$,

$$
\left\langle x^{q}(t)\right\rangle \approx \int_{0}^{\infty} d x F_{t}(x) x^{q}=C_{q} t^{q / 2}
$$

where $C_{q}=2^{q / 2} \Gamma\left(q / 2+d_{s} / 2\right) / \Gamma\left(d_{s} / 2\right)$. The agreement of formula (19) with the numerically computed result is striking, see Fig. 7. considering that there is no free parameters.

We can conclude this section noting that, despite the fractal complexity of $\mathrm{NT}_{k}$, the RW on it does not develop a multiscaling character because its large-scale statistics remains Gaussian-like as clearly indicated by the shape of the approximated distribution (18).

In the next section, we modify the $\mathrm{NT}_{k}$ geometry in order to achieve a RW process with Gaussian scaling of moments $\left\langle|x(t)|^{q}\right\rangle \sim t^{q / 2}$ without Gaussian $\mathrm{PdF}$.

\section{DIFFUSION ON SUPER - BRANCHED GRAPHS}

The structure of the $\mathrm{NT}_{k}$ graph can be easily modified to generate a RW which exhibits standard scaling of all the moments $\left\langle|x(t)|^{q}\right\rangle \sim t^{q / 2}$ without having a Gaussian PdF. We change $N T_{k}$ structure by defining a new type of graph, which we dub $S N T$ (Super Nice Tree). A $S N T$ (Fig. 8) is recursively defined as a $N T_{k}$, but at every branching site $x=2^{n}-1(n=1,2,3, \ldots)$ the tree splits in $k^{n}$ branches. 


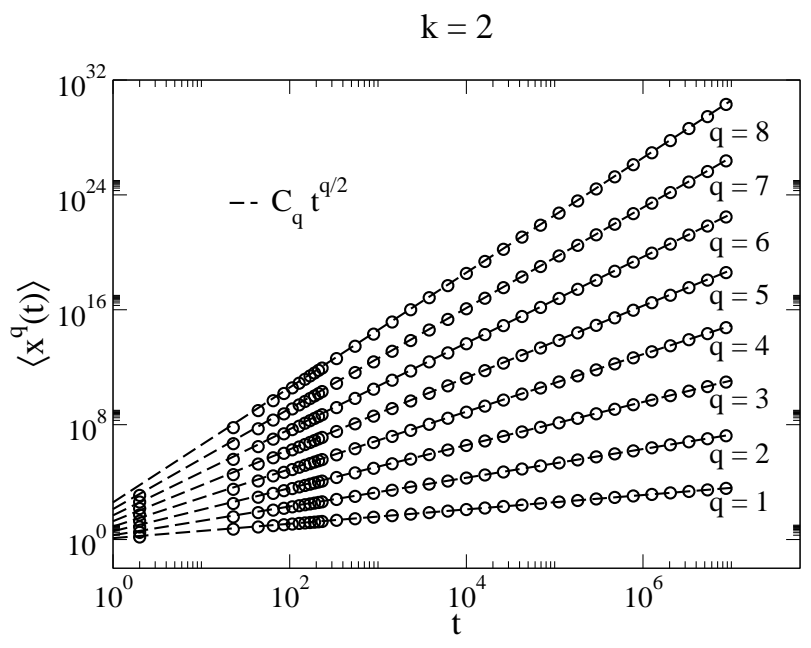

FIG. 7. Time behaviour of the moments, $\left\langle x^{q}(t)\right\rangle(q=1, \ldots, 8)$ obtained by solving numerically the $1 \mathrm{D}$ master equation 15 for a $\mathrm{NT}_{k}$ with $k=2$. All the moments show a Gaussian-like behaviour, which is well explained by the approximated PdF (18). Dashed lines are the results [19.

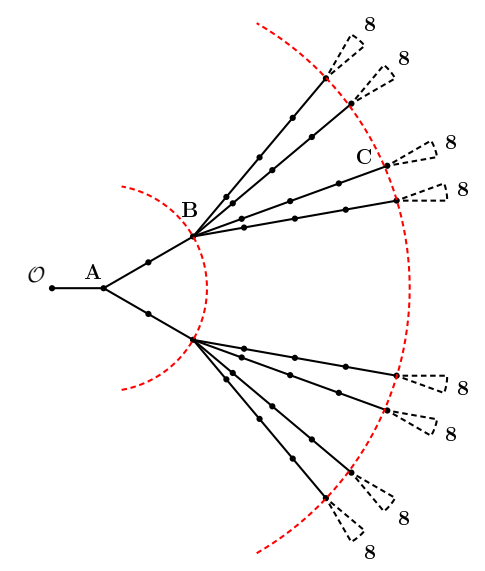

FIG. 8. Sketch of the super branched Nice Tree with $k=2$. It is recursively defined as a $N T_{k}$, but now each branch splits into $k^{n}$ new branches.

In analogy to the $N T_{k}$ case, if we are interested in the process of diffusion from the origin, we again have an equivalence with a random walk on the line with a deterministic distribution of defects (branching points). We have the transition matrix

$$
\begin{aligned}
& w(x+1 \mid x)= \begin{cases}\frac{k^{n}}{k^{n}+1}, & \text { if } x=2^{n}-1 \\
1 / 2, & \text { elsewhere }\end{cases} \\
& w(x-1 \mid x)= \begin{cases}\frac{1}{k^{n}+1}, & \text { if } x=2^{n}-1 \\
1 / 2, & \text { elsewhere }\end{cases}
\end{aligned}
$$

and the corresponding master equation

$$
\begin{aligned}
P_{t+1}(x-1) & =\frac{1}{2} P_{t}(x-2)+\frac{1}{k^{n}+1} P_{t}(x) \\
P_{t+1}(x) & =\frac{1}{2} P_{t}(x-1)+\frac{1}{2} P_{t}(x+1) \\
P_{t+1}(x+1) & =\frac{k^{n}}{k^{n}+1} P_{t}(x)+\frac{1}{2} P_{t}(x+2)
\end{aligned}
$$

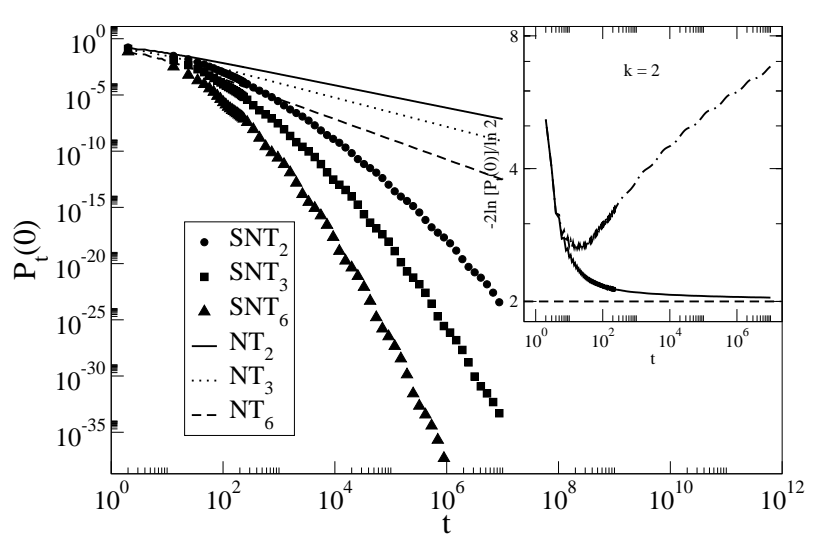

FIG. 9. Probability to come back to the origin in $t$ steps as function of time. The data shows the case of an $S N T$ ( $k=$ $2,3,6)$ compared to the respective cases which one observe for $N T_{k}$. Inset: numerical estimation of the spectral dimension from the asymptotic behaviour of $-2 \ln P_{t}(\mathcal{O}) / \ln 2$ vs. time $t$. The circles refer to a nice tree of dimension $k=2$ which asymptotically approaches the limit $d_{s}=2$; the squares are relative to the super branched case $S N T(k=2)$ showing a non convergence in the explored time range.

The exponential branching of $S N T_{k}$ reduces dramatically the return probability with respect to the $N T_{k}$, Fig. 9. The spectral dimension defined by the return probability scaling (13) seems not to be bounded (inset of Fig. (9). When a RW has traveled on $S N T_{k}$ graph far enough from the origin its return becomes very improbable.

Numerical implementation of the $S N T_{k}$ master equation shows that again moments scale as $\left\langle x^{q}(t)\right\rangle \sim t^{q / 2}$ (see Fig. 10); however the PdF is not a Gaussian and the standard scaling of the PdF at different times fails, see Fig. 11 for the case $k=2$. This is an explicit example where all the positive moments can't identify the probability density (i.e. using moment generating function).

The failure of the standard scaling $x \rightarrow x / \sqrt{\left\langle x^{2}\right\rangle}$, $P_{t}(x) \rightarrow \sqrt{\left\langle x^{2}\right\rangle} P_{t}(x)$ and of the corresponding ordinary property $q \nu(q)=q / 2$ of the moments suggests that there should exist a crossover between two different scaling behaviours in two regions separated by a particular value $\tilde{z}$, such that

$$
P_{t}(x)=\left\{\begin{array}{ll}
h_{t}\left(\frac{x}{\sqrt{t}}\right), & x / \sqrt{t} \leq \tilde{z} \\
\frac{1}{\sqrt{t}} f\left(\frac{x}{\sqrt{t}}\right), & x / \sqrt{t} \geq \tilde{z}
\end{array} .\right.
$$




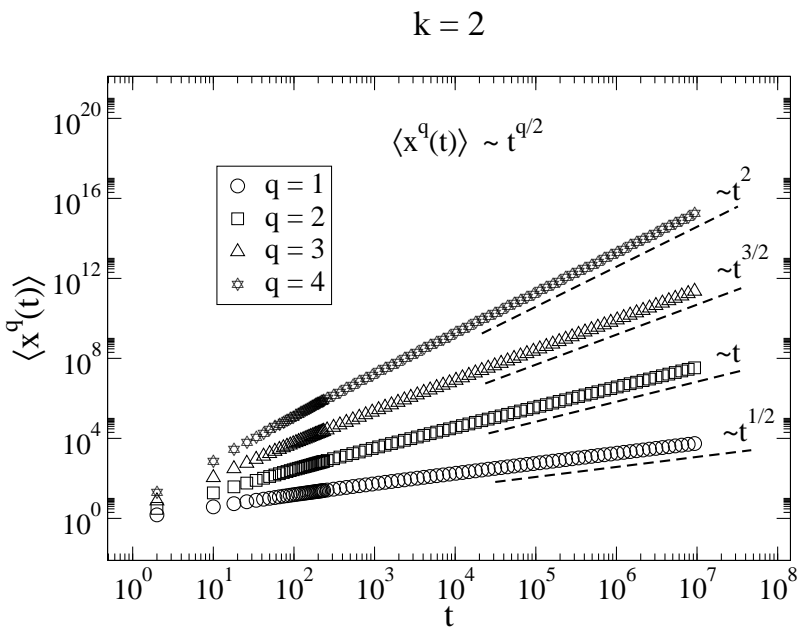

FIG. 10. Time behaviour of four lowest order moments obtained by simulating the $1 \mathrm{D}$ master equation for a $S N T$ with $k=2$; We have also in this case standard diffusion, $\left\langle x^{q}(t)\right\rangle \sim t^{q / 2}$, for all positive $q$, without a Gaussian $P_{t}(x)$.

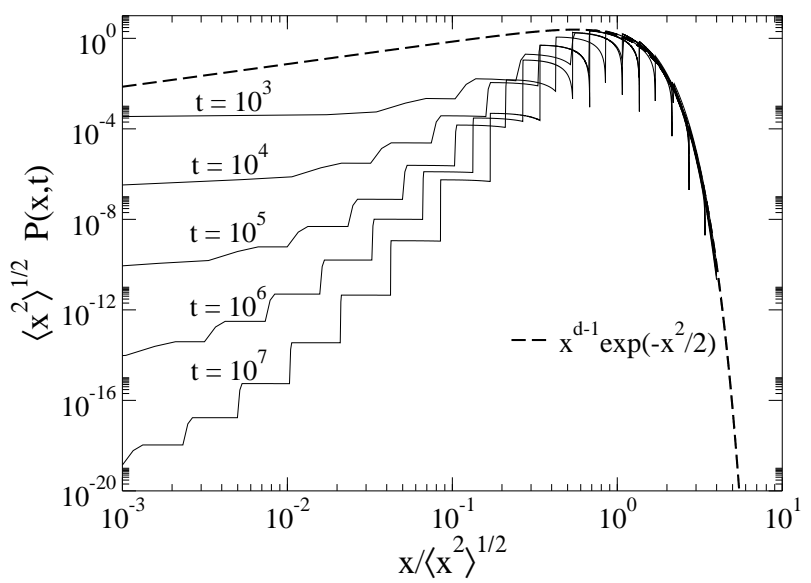

FIG. 11. Probability density at different times for the RW on a $S N T$ graph with $k=2$, generated by the numerical solution of the master equation (22). Unlike the $N T$ case, the lack of collapse after the rescaling indicates that the density of the RW position is not Gaussian at small and intermediate scales. At larger scales, however, the PdF's preserve their Gaussian character as they still collapse to the Gaussian scaling form (18), dashed line.

The above assumptions implies that the moments read

$$
\begin{gathered}
\left\langle x^{q}(t)\right\rangle=\int_{0}^{\sqrt{t} \tilde{z}} d x x^{q} h_{t}(x)+\int_{\sqrt{t} \tilde{z}}^{\infty} d x \frac{x^{q}}{t^{1 / 2}} f\left(\frac{x}{t^{1 / 2}}\right)= \\
\left\langle x^{q}(t)\right\rangle=\int_{0}^{\sqrt{t} \tilde{z}} d x x^{q} h_{t}(x)+A_{q} t^{q / 2}
\end{gathered}
$$

with $A_{q}=\int_{\tilde{z}}^{\infty} d z z^{q} f(z)$ a constant depending on $q$ only. Numerical time behaviour of the $q$-order moments (Fig. 10) is consistent with expression (23) only if the first integral grows more slowly than $t^{q / 2}$.
Since the asymptotic behaviour of the moments implies a Gaussian diffusion but $P_{t}(x)$ is not Gaussian, we could conclude, at a first glance, that some conditions for the applicability of Carleman's theorem [15] on the possibility to reconstruct a $\mathrm{PdF}$ from its moments are violated. Actually the paradox is only apparent, as the Gaussian behaviour $t^{q / 2}$ of the moments becomes exact at sufficiently large $t$. In fact, the standard rescaling $x \rightarrow z=x / \sqrt{\left\langle x^{2}(t)\right\rangle}$ produces the PdF collapse only for $z \gg 1$, while it does not occur at small scales (Fig. 11). This scaling violation at small arguments $z$ follows from the presence of pre-asymptotic terms in Eq. (23).

\section{CONCLUSIONS}

In this paper, we have analyzed the behaviour of the moments and probability distribution of displacements in different random walk models each showing a linear growth of the mean square displacement (MSD). The behaviour $\left\langle x^{2}(t)\right\rangle \sim t$ is generally assumed as a indication of the Fickian or Gaussian diffusion however it can be also consistent with non standard processes.

As an example, we first considered the one-dimensional velocity model of the CTRW which, despite a linear MSD, exhibits multiscaling (strong anomalous diffusion) in higher order moments and distribution.

Moreover, we have analyzed the behaviour of the unbiased random walk on a fractal tree with a branching rate growing exponentially with generations that we termed "super branched graph". Although the diffusion over this graph exhibits a perfect Gaussian property of every positive moment, $\left\langle x^{q}(t)\right\rangle \sim t^{q / 2}$, the Gaussian probability distribution is not granted at every scale.

Conversely, a random walk spanning a "Nice Tree graph", whereby the branching rate grows only linearly with the generation, maintains its large-scale Gaussian diffusion. In this case, the fractal complexity of the tree is unable to destroy the standard behaviour.

The inadequacy of MSD-measurements alone to discriminate between anomalous and normal behaviors has been already discussed in other systems and contexts 29 31], where a standard MSD behaviour coexists with an overall non-Gaussian character of the diffusion. This only apparent contradiction has been termed "paradoxical diffusion" just to stress the peculiarity.

This work supports the view that answering the question "when a Brownian diffusion is standard or anomalous" represents an experimental hard task especially when the systems under observation display a simultaneous statistical and geometrical complexity [3, 20, 29]. This subject has recently regained importance also thank to the advancements of single-molecule experiments which allow the tracking of particle positions with nanoscale resolution. The large amount of high resolution data poses important challenges to the methods of analysis as we can have access to finer statistical properties than the simple MSD behaviour. In this perspective, 
theoretical works similar to the present one may be useful to underscore possible limitations and criticalities in certain straightforward methods of data analysis.

\section{Acknowledgments}

The authors thank prof. R.Burioni for the interesting discussions on graphs and acknowledge the financial support from MIUR, PRIN 2009PYYZM5 "Fluttuazioni: dai sistemi macroscopici alle nanoscale".
[1] A. Einstein, Ann. Phys. 17, 549 (1905)

[2] E. Frey, K. Kroy, Ann. Phys. 14, 20 (2005)

[3] B. Wang, J. Kuo, S.C. Bae, S. Granick, Nature Materials 11, 481 (2012)

[4] R. Klages, G. Radons, I.M. Sokolov, Anomalous transport (Wiley-VCH, 2008)

[5] J.P. Bouchaud, A. Georges, Phys. Rep. 195, 127 (1990)

[6] R. Metzler, J. Klafter, Phys. Rep. 339, 1 (2000)

[7] F. Höfling, T. Franosch, Rep. Prog. Phys. 76, 046602 (2013)

[8] P. Castiglione, A. Mazzino, P. Muratore-Ginanneschi, A. Vulpiani, Physica D 134, 75 (1999)

[9] K. Andersen, P. Castiglione, A. Mazzino, A. Vulpiani, Eur. Phys. J. B 18, 447 (2000)

[10] U. Frisch, M. Vergassola, Europhys. Lett. 14, 439 (1991)

[11] M.H. Jensen, G. Paladin, A. Vulpiani, Phys. Rev. Lett. 67, 208 (1991)

[12] E. Ben-Naim, P.L. Krapivsky, Phys. Rev. E 61, R5 (2000)

[13] M.J. Saxton, K. Jacobson, Annu. Rev. Biophys Biomol. Struc. 26, 373 (1997)

[14] N.I. Akhiezer, N. Kemmer, The classical moment problem: and some related questions in analysis, Vol. 5 (Oliver \& Boyd Edinburgh, 1965)

[15] T. Carleman, C.R. Acad. Sci. Paris 174, 1680 (1922)
[16] E.W. Montroll, G.H. Weiss, J. Math. Phys. 6, 167 (1965)

[17] G.H. Weiss, Aspects and applications of random walks (North-Holland, Amsterdam NL, 1994)

[18] G. Zumofen, J. Klafter, Phys. Rev. E 47, 851 (1993)

[19] R.N. Mantegna, H.E. Stanley, Phys. Rev. Lett. 73, 2946 (1994)

[20] I.M. Sokolov, Soft Matter 8, 9043 (2012)

[21] A. Lubelski, S. I.M., K. J., Phys. Rev. Lett. 100, 250602(1 (2008)

[22] E. Barkai, Y. Garini, R. Metzler, Physics Today 65, 29 (2012)

[23] B. Mandelbrot, The Fractal Geometry of Nature (Freeman, San Francisco USA, 1983)

[24] S. Alexander, R. Orbach, J. Phys. (Paris) Lett. 43, L 625 (1982)

[25] D. ben Avraham, S. Havlin, Diffusion and Reactions in Fractals and Disordered Systems (Cambridge University Press, Cambridge, 2000)

[26] R. Burioni, D. Cassi, Phys. Rev. E 49, R1785 (1994)

[27] R. Burioni, D. Cassi, Phys. Rev. E 51, 2865 (1995)

[28] B.D. Hughes, M. Sahimi, J. Stat. Phys. 29, 781 (1982)

[29] I. Sokolov, J. Mai, A. Blumen, Phys. Rev. Lett. 79, 857 (1997)

[30] I.M. Sokolov, Phys. Rev. E 63, 011104 (2000)

[31] B. Dybiec, E. Gudowska-Nowak, Phys. Rev. E 80, 061122 (2009) 